\title{
Autorinnen und Autoren
}

Acharya, Swati, Prof. Dr., Savitribai Phule Pune University, Department of Foreign Languages, German Section, Ranade Institute Building, Fergusson college Road, Pune 411004, Indien; E-Mail: swarami@gmail.com.

Amann, Wilhelm, Dr., Université du Luxembourg, Institut für deutsche Sprache, Literatur und für Interkulturalität, Maison des Sciences Humaines, 2, avenue de l'Université, 4365 Esch-sur-Alzette, Luxemburg; E-Mail: wilhelm.amann@uni.lu.

Bauer, Matthias, Prof. Dr., Europa-Universität Flensburg, Institut für Sprache, Literatur und Medien, Seminar für Germanistik, Auf dem Campus 1, 24943 Flensburg, Deutschland; E-Mail: matthias.bauer@uni-flensburg.de.

Bremer, Kai, Prof. Dr., Universität Osnabrück, Institut für Kulturgeschichte der Frühen Neuzeit, An der Katharinenkirche 8b, 49074 Osnabrück, Deutschland; E-Mail: kai. bremer@uni-osnabrueck.de.

Costa, Marcella, Prof. Dr., Universitá degli Studi di Torino, Dipartimento di Lingue e Letterature straniere e Culture moderne, Via S. Ottavio, 20, 10124 Torino, Italien; Email: marcella.costa@unito.it

Dembeck, Till, Prof. Dr., Université du Luxembourg, Institut für deutsche Sprache, Literatur und für Interkulturalität, Maison des Sciences Humaines, 2, avenue de l'Université, 4365 Esch-sur-Alzette, Luxemburg; E-Mail: till.dembeck@uni.lu.

Geier, Andrea, Prof. Dr., Universität Trier, FB II Germanistik, Neuere deutsche Literaturwissenschaft, 54286 Trier, Deutschland; E-Mail: geier@uni-trier.de.

Heimböckel, Dieter, Prof. Dr., Université du Luxembourg, Institut für deutsche Sprache, Literatur und für Interkulturalität, Maison des Sciences Humaines, 2, avenue de l'Université, 4365 Esch-sur-Alzette, Luxemburg; E-Mail: dieter.heimboeckel@uni.lu.

Hermes, Stefan, PD Dr., Universität Duisburg-Essen, Fakultät für Geisteswissenschaften, Institut für Germanistik, Universitätsstraße 2, 45141 Essen, Deutschland; E-Mail: stefan.hermes@uni-due.de. 
Holdenried, Michaela, Prof. Dr., Albert-Ludwigs-Universität Freiburg, Deutsches Seminar, Neuere Deutsche Literatur, Platz der Universität 3, 79085 Freiburg, Deutschland; E-Mail: michaela.holdenried@germanistik.uni-freiburg.de

Mein, Georg, Prof. Dr., Université du Luxembourg, Institut für deutsche Sprache, Literatur und für Interkulturalität, Maison des Sciences Humaines, 2, avenue de l'Université, 4365 Esch-sur-Alzette, Luxemburg; E-Mail: georg.mein@uni.lu.

Meyer, Anne-Rose, Prof. Dr., Bergische Universität Wuppertal, Fakultät für Geistesund Kulturwissenschaften, Fachgruppe Germanistik, Gaußstraße 20, 42119 Wuppertal, Deutschland; E-Mail: ameyer@uni-wuppertal.de.

Miguoué, Jean Bertrand, Ass. Prof., Université de Yaoundé I, Département de Langues, Littératures et Civilisations Germaniques, BP. 755, Yaoundé, Kamerun; E-Mail: jb_ miguoue@yahoo.fr.

Patrut, Iulia-Karin, Prof. Dr., Europa-Universität Flensburg, Institut für Sprache, Literatur und Medien, Seminar für Germanistik, Auf dem Campus 1, 24943 Flensburg, Deutschland; E-Mail: iulia-karin.patrut@uni-flensburg.de.

Schiewer, Gesine Lenore, Prof. Dr., Universität Bayreuth, Interkulturelle Germanistik, 95440 Bayreuth, Deutschland; E-Mail: gesine.schiewer@uni-bayreuth.de.

Schößler, Franziska, Prof. Dr., Universität Trier, FB II Germanistik, Neuere deutsche Literaturwissenschaft, 54286 Trier, Deutschland; E-Mail: schoessl@uni-trier.de.

Sieburg, Heinz, Prof. Dr., Université du Luxembourg, Institut für deutsche Sprache, Literatur und für Interkulturalität, Maison des Sciences Humaines, 2, avenue de l'Université, 4365 Esch-sur-Alzette, Luxemburg; E-Mail: heinz.sieburg@uni.lu.

Simo, David, Prof. Dr., Université de Yaoundé I, Département des Langues, Littératures et Civilisations etrangères, Section d'Etudes germaniques, B.P. 755, Yaoundé, Kamerun; E-Mail: simobiegain2o@yahoo.fr.

Teller, Katalin, Dr. habil., Eötvös-Loránd-Universität, Institut für Kunsttheorie und Medienforschung, Lehrstuhl für Ästhetik, Múzeum krt. 6-8, 1088 Budapest, Ungarn; E-Mail:teller.katalin@btk.elte.hu.

Weinberg, Manfred, Prof. Dr., Univerzita Karlova, Filozofická Fakulta, Ústav germánských studií FF UK, Nám. Jana Palacha 2, 11638 Praha 1, Tschechien; E-Mail: Manfred.Weinberg@ff.cuni.cz

Zink, Dominik, Dr., Universität Trier, FB II Germanistik, Neuere deutsche Literaturwissenschaft, Universitätsring 15, 54286 Trier, Deutschland; E-Mail: zinkdo@unitrier.de. 